\title{
A cell factory of Bacillus subtilis engineered for the simple bioconversion of myo-inositol to scyllo-inositol, a potential therapeutic agent for Alzheimer's disease
}

\author{
Masaru Yamaoka ${ }^{1}$, Shin Osawa ${ }^{1}$, Tetsuro Morinaga ${ }^{2}$, Shinji Takenaka ${ }^{1}$ and Ken-ichi Yoshida ${ }^{1{ }^{*}}$
}

\begin{abstract}
Background: A stereoisomer of inositol, scyllo-inositol, is known as a promising therapeutic agent for Alzheimer's disease, since it prevents the accumulation of beta-amyloid deposits, a hallmark of the disease. However, this compound is relatively rare in nature, whereas another stereoisomer of inositol, myo-inositol, is abundantly available.
\end{abstract}

Results: Bacillus subtilis possesses a unique inositol metabolism involving both stereoisomers. We manipulated the inositol metabolism in B. subtilis to permit the possible bioconversion from myo-inositol to scyllo-inositol. Within 48 $\mathrm{h}$ of cultivation, the engineered strain was able to convert almost half of $10 \mathrm{~g} / \mathrm{L}$ myo-inositol to scyllo-inositol that accumulated in the culture medium.

Conclusions: The engineered B. subtilis serves as a prototype of cell factory enabling a novel and inexpensive supply of scyllo-inositol.

\section{Background}

Alzheimer's disease is one of the most common and problematic forms of dementia [1]. In 2006, 26.6 million people suffered from Alzheimer's disease worldwide, and by 2050 , the disease is predicted to increase, affecting 1 in every 85 individuals [2]. Hundreds of clinical trials have been conducted to identify possible treatment strategies for the disease, but despite these efforts no promising strategy has been established. At present, most of the treatment strategies currently used offer only a small symptomatic benefit, and in fact no treatment is available to stop the progression of the disease.

Inositol (1,2,3,4,5,6-cyclohexanehexol) has nine possible stereoisomers due to epimerization of the six hydroxyl groups. The most abundant stereoisomer in nature is myo-inositol (compound 1, Figure 1), which is the structural basis for a number of secondary messengers in eukaryotic cells [3]. In plants, myo-inositol

\footnotetext{
* Correspondence: kenyoshi@kobe-u.ac.jp

${ }^{1}$ Department of Agrobioscience, Graduate School of Agricultural Science,

Kobe University, 1-1 Rokkodai, Nada, Kobe 657 8501, Japan

Full list of author information is available at the end of the article
}

hexakisphosphate or phytic acid often occurs in high bran cereals, serving as the principal storage form of phosphate. This is the main industrial source of myoinositol [4]. The other inositol stereoisomers are relatively rare in nature but some exert specific health-promoting activities. D-chiro-inositol (compound 2) is beneficial for patients with hyperglycemia and polycystic ovary syndrome since it restores insulin sensitivity [5] and recovers normal ovulation [6], respectively. Accumulating evidence indicates that another isomer, scylloinositol (compound 3), has exceptional potential as a therapeutic agent for Alzheimer's disease [7].

scyllo-Inositol is sometimes found in plants and also occurs naturally in human brain as a minor substance that readily crosses the blood-brain barrier [8]. Aggregation of the beta-amyloid peptide in brain cells is a key pathological feature of Alzheimer's disease [9]. scylloInositol directly interacts with the beta-amyloid peptide and blocks the development of fibers, alleviating memory deficits and other symptoms associated with betaamyloid accumulation [10,11]. Furthermore, mice fed with scyllo-inositol have been demonstrated to have

\section{() Biomed Central}


decreased disease symptoms with better cognitive function and greater longevity because the amyloid plaques disappeared and inflammation declined. scyllo-Inositol confers these benefits not only in very young diseasefree mice but also in diseased mice [7]. Thus, scyllo-inositol got fast-track designation from the US Food and Drug Administration for treatment of mild to moderate Alzheimer's disease, and was granted a US patent in 2009 [12]. Phase 2 clinical trials of scyllo-inositol treatment have already been conducted with positive results, and plans for Phase 3 trials have been announced [13].

Bacillus subtilis is one of the best studied Gram-positive bacteria, and its unique inositol metabolism involves at least three of the stereoisomers, myo-inositol, scylloinositol, and D-chiro-inositol (Figure 1) [14]. The B. subtilis iolABCDEFGHIJ operon encodes enzymes involved in multiple steps of inositol metabolism [15]. In the first step, myo-inositol is converted to scyllo-inosose (compound 4, Figure 1) by myo-inositol dehydrogenase, IolG, with $\mathrm{NAD}^{+}$reduction. IolG also dehydrogenates Dchiro-inositol to 1-keto-D-chiro-inositol (compound 5), which is subsequently isomerized by IolI to scyllo-inosose [16]. In the second step, scyllo-inosose is dehydrated by IolE to 3D-(3,5/4)-trihydroxycyclohexane-1,2dione (compound 6) [17]. In the third step, IolD catalyzes the hydrolysis of 3D-(3,5/4)-trihydroxycyclohexane-1,2-dione to yield 5-deoxy-D-glucuronic acid (compound 7). In the next step, IolB catalyzes the isomerization of 5-deoxy-D-glucuronic acid to 2-deoxy-5keto-D-gluconic acid (compound 8), which is subsequently phosphorylated by IolC kinase to 2-deoxy-5keto-D-gluconic acid 6-phosphate (compound 9) [18]. 2Deoxy-5-keto-D-gluconic acid 6-phosphate is an intermediate that acts as an inducer by antagonizing DNA binding of IoIR, a repressor controlling transcription of the $i o l$ operon [19]. Finally, IolJ aldolase catalyzes the conversion of 2-deoxy-5-keto-D-gluconic acid 6-phosphate to dihydroxyacetone phosphate (compound 10) and malonic semialdehyde (compound 11). The former is a glycolytic intermediate and the latter is converted to acetyl-CoA (compound 12), which enters the tricarboxylic acid cycle, and $\mathrm{CO}_{2}$ by the IolA reaction.

B. subtilis possesses two additional and distinct inositol dehydrogenases, IolX and IolW, which specifically act on scyllo-inositol with $\mathrm{NAD}^{+}$and $\mathrm{NADP}^{+}$reduction, respectively [14]. Each of these enzymes convert scylloinositol to scyllo-inosose, which is the same compound produced from myo-inositol by IolG and is readily degraded further via the metabolic pathway described above [18]. iolX transcription is induced after the addition of scyllo-inositol to the growth medium of cells, and its inactivation inhibits cell growth in the presence

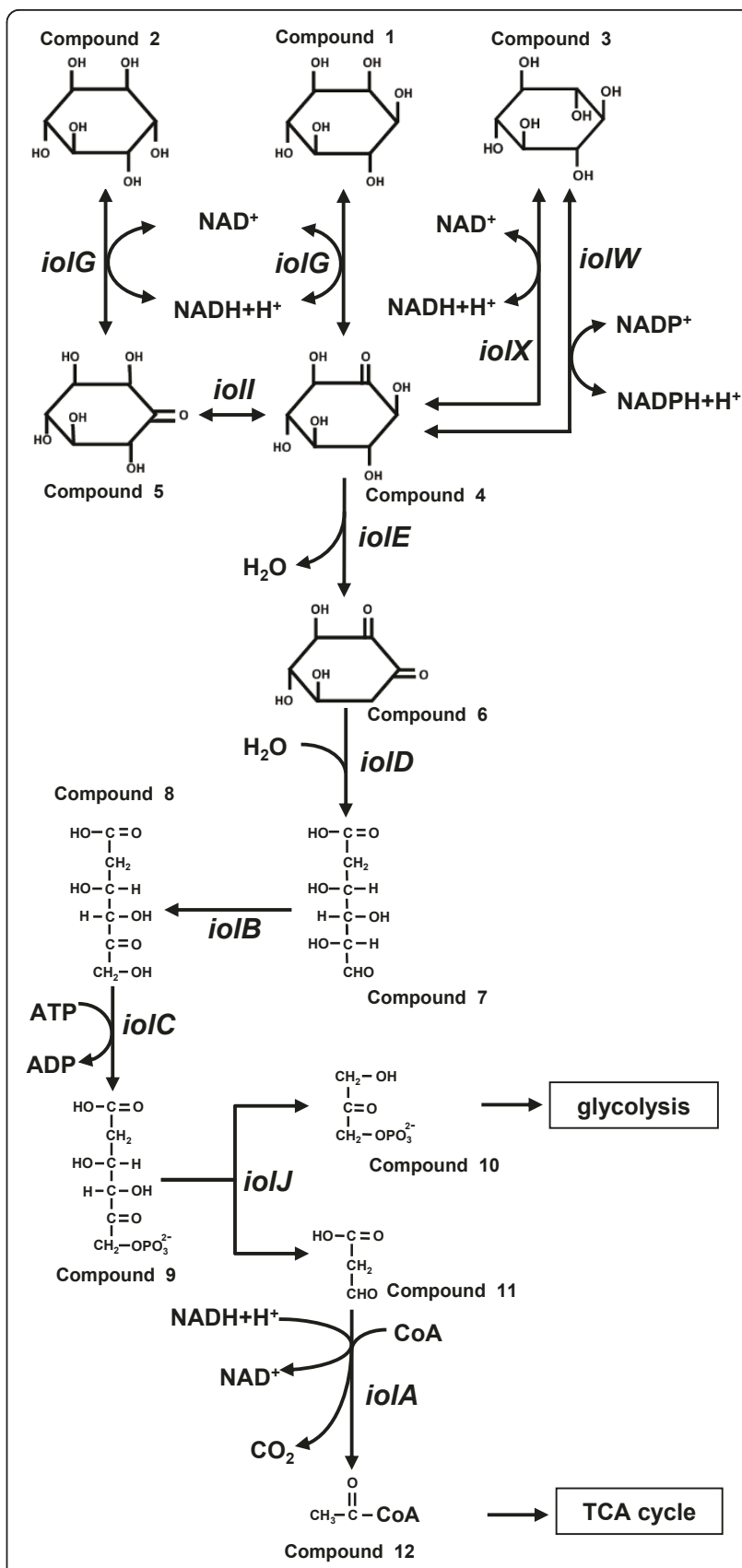

Figure 1 Inositol metabolism in B. subtilis. B. subtilis iol genes encoding enzymes for reactions in the inositol catabolic pathway and the respective intermediate compounds are shown. The intermediate compounds are myo-inositol (compound 1), D-chiroinositol (compound 2), scyllo-inositol I (compound 3), scylloinosose (compound 4), 1-keto-D-chiro-inositol (compound 5), 3D(3,5/4)-trihydroxycyclohexane-1,2-dione (compound 6), 5-deoxy-Dglucuronic acid (compound 7), 2-deoxy-5-keto-D-gluconic acid (compound 8), 2-deoxy-5-keto-D-gluconic acid 6-phosphate (compound 9), dihydroxyacetone phosphate (compound 10), malonic semialdehyde (compound 11), and acetyl-CoA (compound 12). 
of scyllo-inositol as a carbon source, indicating that IolX could function physiologically as a catabolic enzyme [14]. In contrast, iol $W$ transcription is constitutive and its inactivation does not alter the cell growth in the presence of scyllo-inositol. Our previous study suggested that IolW may generate scyllo-inositol from scyllo-inosose with NADPH oxidation, although its physiological role remains unidentified [14].

In this study, we manipulated the inositol metabolism in B. subtilis to control the interconversion among inositol stereoisomers secreted into the culture medium, permitting a simple and inexpensive bioconversion of myo-inositol to scyllo-inositol. The engineered B. subtilis materialized our cell factory concept for scyllo-inositol production.

\section{Results and Discussion}

Analyzing mutations for efficient bioconversion of myoinositol to scyllo-inositol

Previously we demonstrated that B. subtilis strain YF256 (Table 1) was capable of bioconverting myo-inositol to D-chiro-inositol [16]. Because of iolR inactivation [19], strain YF256 constitutively expresses iolG, iolE41, and ioll. The missense mutation allele iolE41 abolishes IolE dehydratase activity, resulting in the intracellular accumulation of scyllo-inosose from myo-inositol due to the IolG reaction [16]. scyllo-Inosose is isomerized by Ioll to 1-keto-D-chiro-inositol and subsequently reduced by IolG to D-chiro-inositol; thus, myo-inositol is converted to D-chiro-inositol, which then appears in the inositol bioconversion medium [16].

In this study, we re-examined the medium in which strain YF256 was cultured for $24 \mathrm{~h}$ by an improved GCTOFMS system [20]. We were surprised to detect not only D-chiro-inositol but also scyllo-inositol (Figure 2A), thus revealing that our previous high-performance liquid chromatography analysis failed to detect scyllo-inositol [16]. Furthermore, this result suggested that some of the accumulated scyllo-inosose could be converted to scylloinositol, probably involving IolX and/or IolW.
When iolI was inactivated (strain TM038, Figure 2B), conversion of scyllo-inosose to D-chiro-inositol decreased to a negligible level, whereas that of scylloinositol increased; however only slightly. These results indicated that we could eliminate the metabolic pathway for D-chiro-inositol by inactivating iolI, although this was not sufficient to increase the conversion to scylloinositol. Therefore, iol $X$ and/or Iol $W$ were inactivated to examine their involvement in scyllo-inositol production. When iolW was functional, iolX inactivation alone drastically elevated the concentration of scyllo-inositol present in the medium (strain TM039, Figure 2C), suggesting that IolX may degrade scyllo-inositol. In contrast, IolW was essential for efficient scyllo-inositol production (strains TM040 and TM041, Figure 2D and 2E, respectively). These results clearly indicate that IolW is crucial for the conversion of scyllo-inosose to scylloinositol.

\section{Properties of the scyllo-inositol producing B. subtilis cell factory}

After 48-h culture of strain TM039 in the inositol bioconversion medium (Figure 3A), almost half of the myoinositol was converted to scyllo-inositol. This simple bioconversion was so successful that it realized our concept of cell factory to produce scyllo-inositol with higher translational potential in the yield and purity of product than the other methods previously described [21,22]. Further culture resulted in the consumption of not only myo-inositol but also some of the scyllo-inositol, and particularly myo-inositol was consumed almost completely after $96 \mathrm{~h}$ of culture whereas scyllo-inositol remained relatively intact. The results implied that the mutated IolE41 enzyme, which did not support cell growth using myo-inositol as the carbon source [17], might possibly retain a certain lower level of scyllo-inosose degrading activity, which resulted in the decrease in the two inositol isomers; because myo-inositol is preferentially converted to scyllo-inosose by IolG reaction, it decreased more obviously. Although we do not have

Table 1 Bacterial strains used in this study.

\begin{tabular}{lll}
\hline Strain & Relevant genotype & Source or reference \\
\hline 60015 & Wild-type & Laboratory stock \\
BFS3018 & iolX::pMutin2(erm) & National Institute of Genetics [14] \\
FU341 & asnH::spc & {$[24]$} \\
TM038 & iolE41 ioll::spc iolR::cat & This work \\
TM039 & iolE41 ioll::spc iolR::cat iolX::pMutin4(erm) & This work \\
TM040 & iolE41 ioll:::spc iolR::cat iolW::pMutin2(erm::tet) & This work \\
TM041 & iolE41 ioll::spc iolR::cat iolW::pMutin2(erm::tet) iolX::pMutin4(erm) & This work \\
TM043 & iolW::pMutin2(erm::tet) & {$[14]$} \\
YF256 & iolE41 iolR::cat & {$[17]$} \\
\hline
\end{tabular}

The integration vector pMutin2 originally possesses the erm marker [26], which was replaced with the tet marker in TM043 as described previously [23]. 


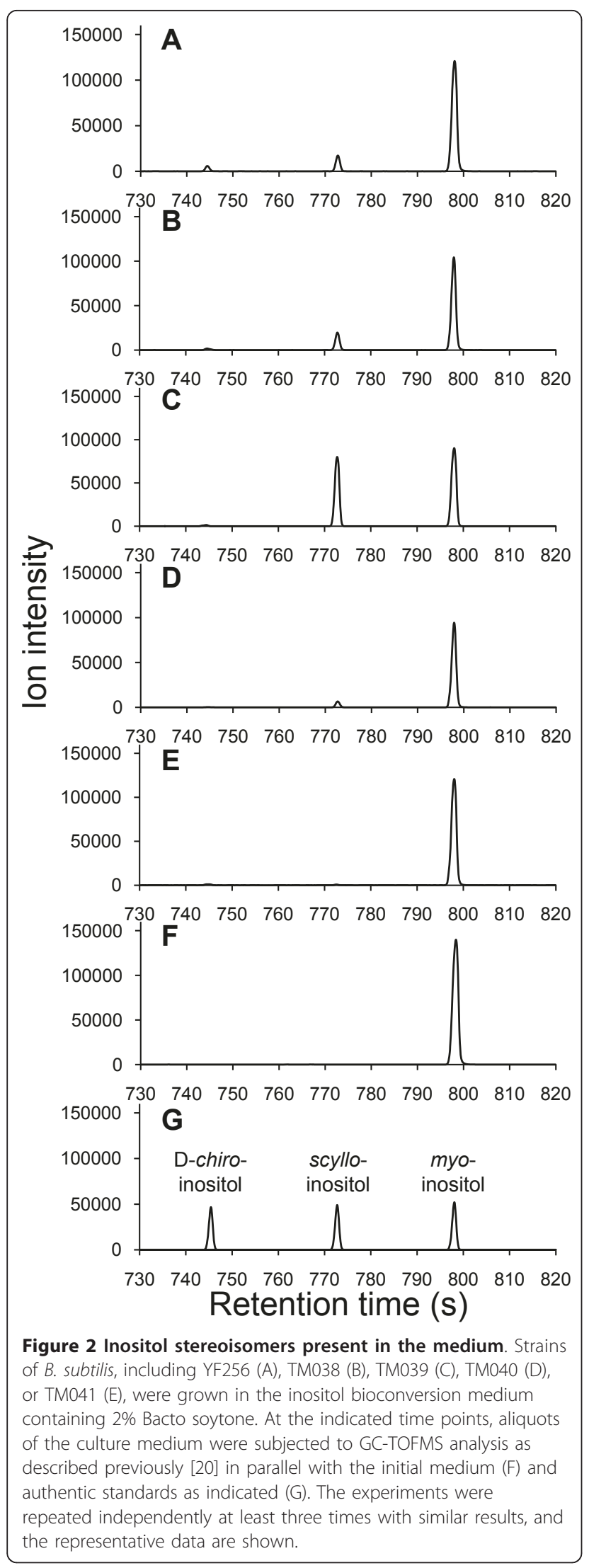

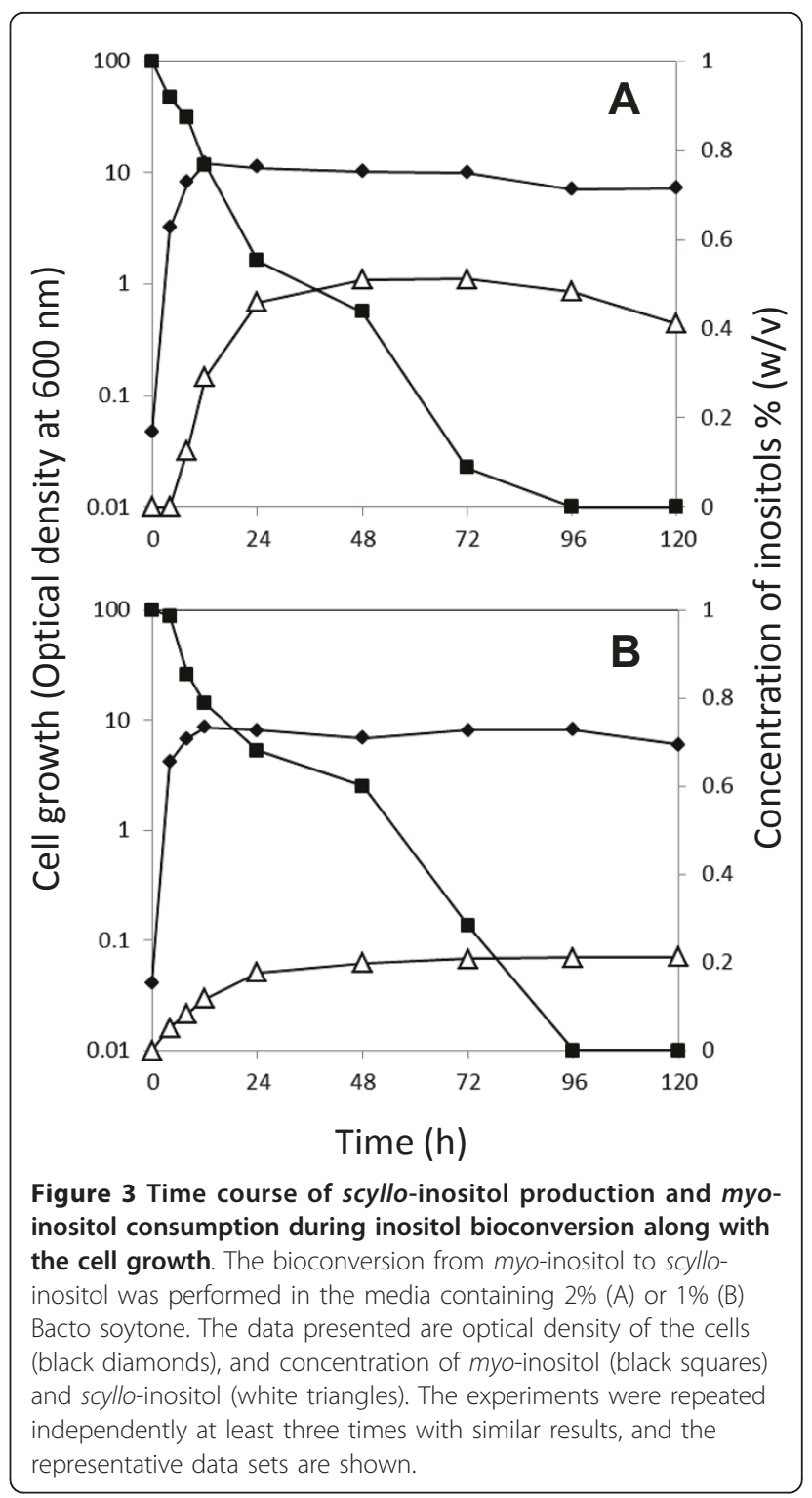

experimental evidences to prove the possibility mentioned above, nevertheless the selective consumption of myo-inositol could be even practical for a cell factory making the scyllo-inositol product free from significant myo-inositol contamination. However, to avoid wastage of both the material and the product, possible strategies to stop the inositol degradation will be considered, including in-frame deletion of iolE.

In principal, we could assume that IolG reaction is due to a lowering of the intracellular $\mathrm{NAD}^{+} / \mathrm{NADH}$ ratio, whereas the IolW reaction elevates the NADP +/NADPH ratio (Figure 1). If this is actually the case, the inevitable problem of cofactor imbalance must have been induced in the cells. Although considerably efficient bioconversion has already been achieved (Figure 
3A), it may be improved by correcting the possible cofactor imbalance under the given conditions. Interestingly, when the major nutrient (Bacto soytone) contained in the conversion medium was reduced by half, no severe effect on the cell growth was observed, nevertheless only scyllo-inositol production was impaired significantly while myo-inositol was consumed almost completely (Figure 3B). The results imply that there might be some nutritional and/or metabolic factors limiting the bioconversion, which should be identified and then controlled in the next generation of realistic cell factory with optimized efficiency in scyllo-inositol production.

It was demonstrated that $B$. subtilis possesses two distinct inositol transporters, IolT and IolF, which are different in substrate specificity to myo-inositol and Dchiro-inositol [23]. Our preliminary observations suggested that scyllo-inositol transport into the cells could mainly depend on IolT, which is similar to myo-inositol (data not shown), and overexpression of iolT may not be effective for improving the conversion efficiency. However, it has not yet been explained how the inositol stereoisomers are secreted into the medium after conversion; hence, we speculate the involvement of an unknown efflux pump, which is also currently being investigated as one of the possible targets to be engineered.

\section{Conclusions}

B. subtilis is one of the best studied gram-positive bacteria. Its unique inositol metabolism has been completely elucidated to involve at least three inositol stereoisomers, including scyllo-inositol and myo-inositol. Our previous knowledge and findings suggested possible interconversion among the stereoisomers. Therefore, we manipulated the metabolic pathway to realize a cell factory concept enabling the simple and inexpensive bioconversion of myo-inositol to scyllo-inositol, which is a promising therapeutic agent for Alzheimer's disease.

\section{Methods}

\section{Bacterial strains and their constructions}

Bacterial strains used in this study are listed in Table 1. The iolI gene of strain YF256 was inactivated by inserting a spectinomycin resistance gene $(s p c)$ cassette as follows. A DNA fragment carrying the $s p c$ cassette was amplified from strain FU341 DNA [24] by PCR using IolI3F and IolI4R primer pair (Table 2). Further, two DNA fragments corresponding to the upstream and downstream regions of iolI were amplified from strain 60015 DNA by PCR using IolI1F/IolI2R and IolI5F/ Iol6R primer pairs, respectively, (Table 2 ). The 5 ' end sequences of IolI3F and Iol4R were complementary to IolI2R and IolI5F, respectively. The three PCR fragments
Table 2 Oligonucleotide primers used in this study

\begin{tabular}{ll}
\hline Primer & Sequence \\
\hline ioll1F & 5'-TGCGGTTGAACTTGAAGTGG-3' \\
ioll2R & 5'-TCTTCTGCTCTGTCACAAGC-3' \\
ioll3F & 5'-GCTTGTGACAGAGCAGAAGACAATAACGCTATTGGGAG-3' \\
ioll4R & 5'-GAACCCATTGCATGGAAGTGCTATATGCTCCTTCTGGC-3' \\
ioll5F & 5'-CACTTCCATGCAATGGGTTC-3' \\
ioll6R & 5'-ATATTGATCTTCGCGTGGCC-3' \\
\hline
\end{tabular}

were mixed and ligated using PCR performed with the outside primer pair IolIF1/Iol6R. The resulting PCR fragment was used to transform strain YF256 to obtain strain TM038 with spectinomycin resistance (iolI::spc; Table 1).

Strain TM038 was transformed with strain BFS3018 DNA [14] to obtain strain TM039 with erythromycin resistance (iolX::pMutin4(erm); Table 1) and with strain TM043 DNA [14] to obtain strain TM040 with tetracycline resistance (iolW::pMutin2(erm::tet); Table 1). Strain TM040 was further transformed with strain BFS3018 DNA to obtain strain TM041 with erythromycin resistance (iolW::pMutin2(erm::tet) iolX::pMutin4(erm); Table 1).

\section{Culture conditions}

Bacterial strains were maintained in Luria-Bertani medium [25]. For inositol bioconversion, a 500-ml Sakaguchi flask with a breathable foam stopper, containing $30 \mathrm{ml}$ of inositol bioconversion medium, containing $2 \%$ or $1 \%$ (w/v) Bacto soytone (Becton, Dickinson and Co., Sparks, MD), 0.5\% Bacto yeast extract (Becton, Dickinson and Co.), $0.5 \% \mathrm{NaCl}$, and $1 \%$ myo-inositol, was inoculated with one of the B. subtilis strains at an optical density of 0.05 at $600 \mathrm{~nm}$, and incubated at $37^{\circ} \mathrm{C}$ with shaking at $150 \mathrm{rpm}$.

\section{Measurement of inositol stereoisomers in the medium}

After bioconversion, aliquots of the culture medium were subjected to gas chromatography-time-of-flight mass spectrometry (GC-TOFMS) analysis as described [20] but with some modifications as follows. After removing bacterial cells by centrifugation, an aliquot of the culture medium was evaporated in a test tube under vacuum. The dried pellet was dissolved in the extraction mixture of water, chloroform, and methanol (2:2:5), and incubated at $150^{\circ} \mathrm{C}$ for $30 \mathrm{~min}$ with vigorous shaking. After centrifugation at $40^{\circ} \mathrm{C}$ for $3 \mathrm{~min}$ at $16,000 \mathrm{~g}$, the supernatant was transferred to another tube and diluted appropriately with pure water. After another centrifugation, part of the supernatant was dried completely in another tube. The dried pellet was dissolved, and the substances present in it were derivatized with methoxyamine hydrochloride in pyridine at $30^{\circ} \mathrm{C}$ for $90 \mathrm{~min}$ with 
vigorous shaking. After the addition of $\mathrm{N}$-methyl- $\mathrm{N}$-(trimethylsilyl) trifluoroacetamide (GL Science, Tokyo, Japan), the derivatized substances were further incubated at $37^{\circ} \mathrm{C}$ for $30 \mathrm{~min}$, and then injected via an Agilent 7683B autosampler into an Agilent 7890A GC (Agilent Technologies, Palo Alto, CA) coupled to a Pegasus HT TOFMS (Leco Corp., St Joseph, MI) with the settings as described [20]. Peak deconvolution identification and quantification were performed using the Pegasus ChromaTOF software package ver. 4.21 (Leco Corp.). Commercially available authentic inositols were derivatized and analyzed in parallel with the experimental samples. The mass spectra and retention time obtained were used to identify the stereoisomers, and ion intensities were used to calculate concentration of the stereoisomers.

\section{Acknowledgements}

We thank Dr. Tomohisa Hasunuma, Mr. Kazuya Yoshimura, and Prof. Akihiko Kondo for their indispensable advice regarding GC-TOFMS analysis as well as their continuous support. Strain BFS3018 was supplied by the National Institute of Genetics, Japan, within the framework of the National BioResource Project: E. coli and B. subtilis. This work was supported in part by KAKENHI (22310130), Special Coordination Funds for Promoting Science and Technology, Creation of Innovative Centers for Advanced Interdisciplinary Research Areas, and Advanced Low Carbon Technology Research and Development Program, MEXT, Japan.

\section{Author details}

'Department of Agrobioscience, Graduate School of Agricultural Science, Kobe University, 1-1 Rokkodai, Nada, Kobe 657 8501, Japan. ${ }^{2}$ Process Engineering Department, Manufacturing Management Division, Sysmex Corporation, 4-4-4 Takatsukadai, Nishi-ku, Kobe 651-2271, Japan.

\section{Authors' contributions}

All authors read and approved the manuscript. MY, SO, and TM equally contributed to experiments. ST was involved in data analysis and interpretation. KY designed and conducted this research project as the principal investigator, being also responsible for manuscript preparation.

\section{Competing interests}

The content of this manuscript is relevant to a patent application made by Kobe University (PCT/JP2009/005782), but all authors declare that they have no competing interests.

Received: 26 July 2011 Accepted: 7 September 2011

Published: 7 September 2011

\section{References}

1. What is Alzheimer's disease?. [http://www.alzheimers.org.uk/site/scripts/ documents_info.php?document|D=100].

2. Brookmeyer R, Johnson E, Ziegler-Graham K, Arrighi HM: Forecasting the global burden of Alzheimer's disease. Alzheimers Dement 2007, 3:186-191.

3. Irvine RF, Schell MJ: Back in the water: the return of the inositol phosphates. Nat Rev Mol Cell Biol 2001, 2:327-338.

4. Reddy NR, Sathe SK, Salunkhe DK: Phytates in legumes and cereals. Adv Food Res 1982, 28:1-92.

5. Larner J: D-chiro-inositol-its functional role in insulin action and its deficit in insulin resistance. Int J Exp Diabetes Res 2002, 3:47-60.

6. Nestler JE, Jakubowicz DJ, Reamer P, Gunn RD, Allan G: Ovulatory and metabolic effects of D-chiro-inositol in the polycystic ovary syndrome. N Engl J Med 1999, 340:1314-1320.

7. McLaurin J, Kierstead ME, Brown ME, Hawkes CA, Lambermon MH, Phinney AL, Darabie AA, Cousins JE, French JE, Lan MF, Chen F, Wong SS, Mount HT, Fraser PE, Westaway D, St George-Hyslop P: Cyclohexanehexol inhibitors of Abeta aggregation prevent and reverse Alzheimer phenotype in a mouse model. Nat Med 2006, 12:801-808.

8. Spector R: The transport and metabolism of scyllo-inositol in the central nervous system. J Neurochem 1978, 31:1113-1115.

9. Geylis V, Steinitz M: Immunotherapy of Alzheimer's disease (AD): from murine models to anti-amyloid beta (Abeta) human monoclonal antibodies. Autoimmun Rev 2006, 5:33-39.

10. McLaurin J, Golomb R, Jurewicz A, Antel JP, Fraser PE: Inositol stereoisomers stabilize an oligomeric aggregate of Alzheimer amyloid beta peptide and inhibit abeta-induced toxicity. J Biol Chem 2000, 275:18495-18502.

11. Hawkes CA, Deng LH, Shaw JE, Nitz M, McLaurin J: Small molecule betaamyloid inhibitors that stabilize protofibrillar structures in vitro improve cognition and pathology in a mouse model of Alzheimer's disease. Eur J Neurosci 2010, 31:203-213.

12. McLaurin J: Methods of preventing, treating and diagnosing disorders of protein aggregation.[http://www.freepatentsonline.com/7521481.pdf].

13. Anti-A $\beta$ Oligomer Headed for Phase 3 Clinical Trial. [http://www.alzforum. org/new/detail.asp?id=2530].

14. Morinaga T, Ashida H, Yoshida K: Identification of two scyllo-inositol dehydrogenases in Bacillus subtilis. Microbiology 2010, 156:1538-1546.

15. Yoshida K, Aoyama D, Ishio I, Shibayama T, Fujita Y: Organization and transcription of the myo-inositol operon, iol, of Bacillus subtilis. J Bacteriol 1997, 179:4591-4598

16. Yoshida K, Yamaguchi M, Morinaga T, Ikeuchi M, Kinehara M, Ashida H: Genetic modification of Bacillus subtilis for production of D-chiro-inositol, an investigational drug candidate for treatment of type 2 diabetes and polycystic ovary syndrome. Appl Environ Microbiol 2006, 72:1310-1315.

17. Yoshida K, Yamaguchi M, Ikeda H, Omae K, Tsurusaki K, Fujita Y: The fifth gene of the iol operon of Bacillus subtilis, iolE, encodes 2-keto-myoinositol dehydratase. Microbiology 2004, 150:571-580.

18. Yoshida K, Yamaguchi M, Morinaga T, Kinehara M, Ikeuchi M, Ashida H, Fujita Y: myo-Inositol catabolism in Bacillus subtilis. J Biol Chem 2008, 283:10415-10424.

19. Yoshida K, Shibayama T, Aoyama D, Fujita Y: Interaction of a repressor and its binding sites for regulation of the Bacillus subtilis iol divergon. $J \mathrm{Mol}$ Biol 1999, 285:917-929.

20. Hasunuma T, Sanda T, Yamada R, Yoshimura K, Ishii J, Kondo A: Metabolic pathway engineering based on metabolomics confers acetic and formic acid tolerance to a recombinant xylose-fermenting strain of Saccharomyces cerevisiae. Microb Cell Fact 2011, 10:2.

21. Hipps PP, Holland WH, Sherman WR: Interconversion of myo- and scylloinositol with simultaneous formation of neo-inositol by an NADP ${ }^{+}$ dependent epimerase from bovine brain. Biochem Biophys Res Commun 1977, 77:340-346.

22. Yamaguchi M, Kita Y, Mori T, Kanbe K, Tomoda A, Takahashi A, Ichikawa W: Method for producing scyllo-inositol, United States Patent US7745671. [http://www.freepatentsonline.com/7745671.pdf].

23. Morinaga $T$, Matsuse $T$, Ashida $H$, Yoshida K: Differential substrate specificity of two inositol transporters of Bacillus subtilis. Biosci Biotechnol Biochem 2010, 74:1312-1314.

24. Yoshida K, Fujita Y, Ehrlich SD: Three asparagine synthetase genes of Bacillus subtilis. J Bacteriol 1999, 181:6081-6091.

25. Sambrook J, Russell DW: Molecular Cloning: A Laboratory Manual Cold Spring Harbor, Cold Spring Harbor Press; 2001.

26. Vagner $\mathrm{V}$, Dervyn $\mathrm{E}$, Ehrlich SD: A vector for systematic gene inactivation in Bacillus subtilis. Microbiology 1998, 144:3097-3104.

doi:10.1186/1475-2859-10-69

Cite this article as: Yamaoka et al: A cell factory of Bacillus subtilis engineered for the simple bioconversion of myo-inositol to scylloinositol, a potential therapeutic agent for Alzheimer's disease. Microbial Cell Factories 2011 10:69. 